\title{
ON THE ANNULUS CONJECTURE ${ }^{1}$
}

\author{
R. C. KIRBY
}

The annulus conjecture states that the closure of the region between two locally flat $(n-1)$-spheres in $R^{n}$ is homeomorphic to $S^{n-1} \times[-1,1]$. The annulus conjecture is fundamental to geometric topology (see [5, p. 579]); for example its truth in dimensions $\leqq k$ implies that any homeomorphism of $R^{k}$ is stable [3]. We will prove here a weakened form of this conjecture (Theorem 1 ).

An imbedding $f: S^{n-1} \rightarrow R^{n}$ is said to be locally flat if each point $p \in S^{n-1}$ has a neighborhood $N$ such that $f$ extends to an imbedding $f: N \times[-1,1] \rightarrow R^{n}$ with $N \times 0$ identified with $N . f$ is called flat or bicollared if it extends to an imbedding $f: S^{n-1} \times[-1,1] \rightarrow R^{n}$. Brown [1] proved that a locally flat imbedding is bicollared.

An imbedding $f: S^{n-1} \times[-1,1] \rightarrow R^{n}$ is said to be somewhere planar if there exist $p \in S^{n-1}, q \in(-1,1)$, a neighborhood $N$ of $(p, q)$ in $S^{n-1} \times q$, and an $(n-1)$-plane $P$ in $R^{n}$ such that $f(N)$ projects homeomorphically into $P$ along normals to $P$.

$f$ is clearly somewhere planar if it is piecewise linear on some open set of $S^{n-1} \times q, q \in(-1,1)$. According to Corollary $7, f$ is also somewhere planar if it is differentiable with nonzero Jacobian at some point $(p, q) \in S^{n-1} \times(-1,1)$.

It is with the added condition that each imbedding is somewhere planar that we prove the then weakened annulus conjecture. If the imbeddings are differentiable or piecewise linear, then it is already known that the annulus conjecture holds for $n \geqq 6$ using the $h$ cobordism theorems of [7] and [6].

THEOREM 1. Let $f, g: S^{n-1} \times[-1,1] \rightarrow R^{n}$ be two imbeddings with disjoint images such that $f$ and $g$ are both somewhere planar. Then the closure of the region between $f\left(S^{n-1} \times 0\right)$ and $g\left(S^{n-1} \times 0\right)$ is homeomorphic to $S^{n-1} \times[-1,1]$.

The proof will follow from Theorems 2 and 5 . The basic idea is that the region between the two $(n-1)$-spheres minus a tube $T$ joining the spheres is bounded by a locally flat $(n-1)$-sphere and is thus an $n$-ball by the Schoenflies theorem (see [2]). See Figure 1.

In the next theorem, we assume the existence of this tube and then

Received by the editors July 6, 1964 .

1 The contents of this paper form part of the author's dissertation at the University of Chicago written under Professor Eldon Dyer. 
obtain an annulus, and in Theorem 5 we show that the existence of the tube follows from the somewhere planar conditions.

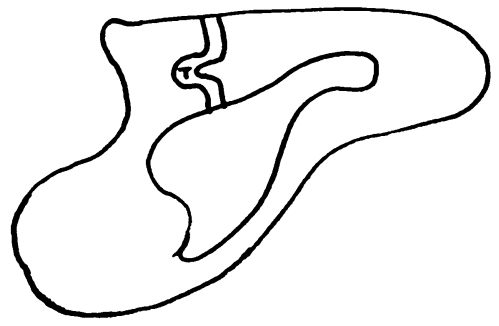

FIGURE 1

TheOREM 2. Let $f, g: S^{n-1} \times[-1,1] \rightarrow R^{n}$ be two imbeddings with disjoint images. Let $h: B^{n-1} \times[-1,1] \rightarrow R^{n}$ be an imbedding of the unit $(n-1)$-ball satisfying

(i) $h\left(B^{n-1} \times-1\right) \subset f\left(S^{n-1} \times q\right)$ for $q \in(-1,1)$,

(ii) $h\left(B^{n-1} \times 1\right) \subset g\left(S^{n-1} \times q^{\prime}\right)$ for $q^{\prime} \in(-1,1)$,

(iii) $h\left(B^{n-1} \times(-1,1)\right)$ is a subset of the open region between $f\left(S^{n-1} \times q\right)$ and $g\left(S^{n-1} \times q^{\prime}\right)$.

Then there exists a homeomorphism $A: S^{n-1} \times[-1,1] \rightarrow \bar{Z}$ where $\bar{Z}$ is equal to the closure of the region $Z$ between $f\left(S^{n-1} \times 0\right)$ and $g\left(S^{n-1} \times 0\right)$.

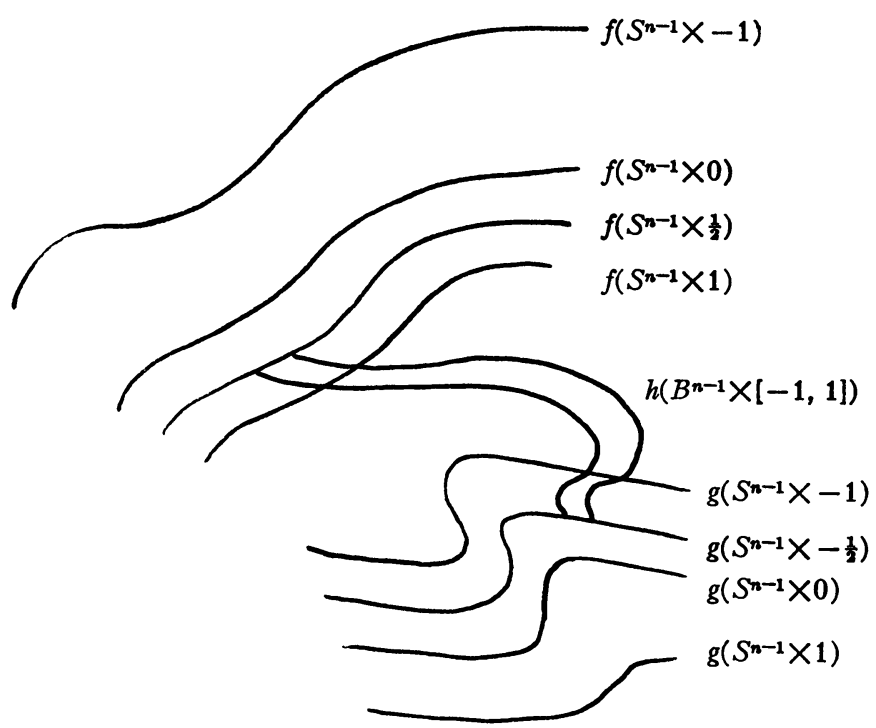

Figure 2 
Lemma 3. Let $f, g$ be as above. Suppose B maps $S^{n-1} \times[-1,1]$ homeomorphically onto the closure of the region between $f\left(S^{n-1} \times t\right)$ and $g\left(S^{n-1} \times t^{\prime}\right), t, t^{\prime} \in(-1,1)$. Then there exists a map $A$, mapping $S^{n-1} \times[-1,1]$ homeomorphically onto $\bar{Z}$.

Proof. Let $k$ be the obvious homeomorphism of $R^{n}$ moving $f\left(S^{n-1} \times t\right)$ to $f\left(S^{n-1} \times 0\right)$ and fixing points outside $f\left(S^{n-1} \times[-1,1]\right)$. Define $k^{\prime}$ similarly, so that it moves $g\left(S^{n-1} \times t^{\prime}\right)$ to $g\left(S^{n-1} \times 0\right)$ and fixes $R^{n}-g\left(S^{n-1} \times[-1,1]\right)$. Then $A=k^{\prime} k B$ satisfies the lemma.

Proof OF THEOREM 2.

STEP 1. We will prove the theorem for $q=\frac{1}{2}, q^{\prime}=-\frac{1}{2}$. The same method would show that, for general $q$ and $q^{\prime}$, the closure of the region between $f\left(S^{n-1} \times t\right), t \in(-1, q)$ and $g\left(S^{n-1} \times t^{\prime}\right), t^{\prime} \in\left(q^{\prime}, 1\right)$ is homeomorphic (by a homeomorphism $B$ ) to an annulus. Thus by Lemma 3, $\bar{Z}$ would be homeomorphic to an annulus. See Figure 2 .

STEP 2. $h\left(B^{n-1} \times[-1,1]\right)$ is a solid tube with one end, $h\left(B^{n-1} \times-1\right)$, lying homeomorphically in $f\left(S^{n-1} \times \frac{1}{2}\right)$. We can lengthen this tube so that this end lies in $f\left(S^{n-1} \times 0\right)$ by extending it along the "collaring of $f^{\prime \prime}$ (see Figure 3).

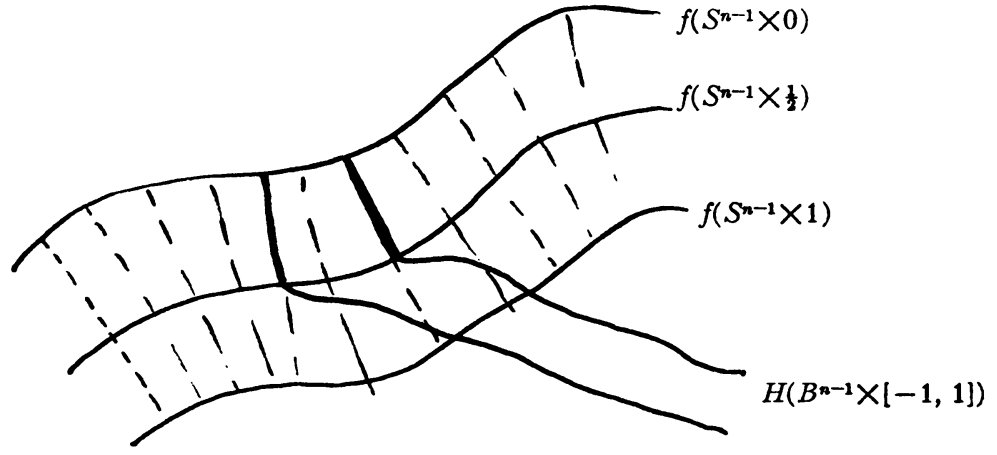

Figure 3

Let $h^{\prime}: B^{n-1} \times[-1,1] \rightarrow R^{n}$ be defined by

$$
h^{\prime}(x, t)= \begin{cases}f k_{t} f^{-1} h(x,-1) & \text { for }-1 \leqq t \leqq-\frac{1}{2}, \\ h(x, 2 t) & \text { for }-\frac{1}{2} \leqq t \leqq 0, \\ h(x, t) & \text { for } 0 \leqq t \leqq 1,\end{cases}
$$

where $k_{t}: S^{n-1} \times \frac{1}{2} \rightarrow S^{n-1} \times(1+t)$ by $k_{t}\left(y, \frac{1}{2}\right)=(y, 1+t)$, for $-1 \leqq t$ $\leqq-\frac{1}{2}$. Since $k_{-1 / 2}=$ identity, $h^{\prime}\left(x,-\frac{1}{2}\right)$ is well defined. $k_{-1}\left(y, \frac{1}{2}\right)$ $=(y, 0)$ so $h^{\prime}\left(B^{n-1} \times-1\right) \subset f\left(S^{n-1} \times 0\right)$. The last part of the tube now 
agrees with the collaring of $f\left(S^{n-1} \times 0\right)$ due to $f$. We can proceed in the same way with the other end of the tube. Let $H$ denote the final homeomorphism $H: B^{n-1} \times[-1,1] \rightarrow R^{n}$.

STEP 3. $H\left(\partial B_{3}^{n-1} \times-1\right)$ is a bicollared imbedding of $S^{n-2}$ in $S^{n-1}$ $=f\left(S^{n-1} \times 0\right)$. According to the Schoenflies theorem [2], there exists a homeomorphism $a: B_{i}^{n-1} \rightarrow f\left(S^{n-1} \times 0\right)-H$ (int $B_{i}^{n-1} \times-1$ ) where $B_{r}^{k}$ denotes the $k$-ball of radius $r$. Extend $a$ to $a^{\prime}: B_{j}^{n-1} \times[-1,1] \rightarrow R^{n}$ by $a^{\prime}(x, s)=f j_{s} f^{-1} a(x)$, where $j_{s}: S^{n-1} \times 0 \rightarrow S^{n-1} \times s$ is defined by $j_{s}(y, 0)=(y, s)$. We note that $a^{\prime}(y \times[0,1]) \subset H(y \times[-1,1])$ for $y \in \partial B_{i}^{n-1}$. We can similarly obtain $b$ and $b^{\prime}$ for $g$. This step was necessary to give $f\left(S^{n-1} \times 0\right)-H\left(\right.$ int $\left.B_{3}^{n-1} \times-1\right)$ the coordinates of an $(n-1)$-ball.

STEP 4. Considering $S^{n-1}$ as the unit $(n-1)$-sphere in $R^{n}$, define the subsets

$$
\begin{aligned}
L & =\left\{x \in S^{n-1} \mid \frac{1}{2} \leqq x_{n}\right\}, \\
M & =\left\{x \in S^{n-1} \mid-\frac{1}{2} \leqq x_{n} \leqq \frac{1}{2}\right\}, \\
N & =\left\{x \in S^{n-1} \mid x_{n} \leqq-\frac{1}{2}\right\} .
\end{aligned}
$$

We define the natural homeomorphisms $k: L \rightarrow B_{3}^{n-1}, k^{\prime}: M \rightarrow \partial B_{3}^{n-1}$ $\times[-1,1]$, and $k^{\prime \prime}: N \rightarrow B_{1}^{n-1}$, so that $k^{\prime}(x)=(k(x),-1)$ for $x \in L \cap M$, and $k^{\prime}(x)=\left(k^{\prime \prime}(x), 1\right)$ for $x \in M \cap N$. Then we construct an imbedding $c: S^{n-1}=L \cup M \cup N \rightarrow R^{n}$ defined by $c\left|L=a^{\prime} k, c\right| M=H k^{\prime}$, and $c \mid N$ $=b^{\prime} k^{\prime \prime}$. See Figure 4 .

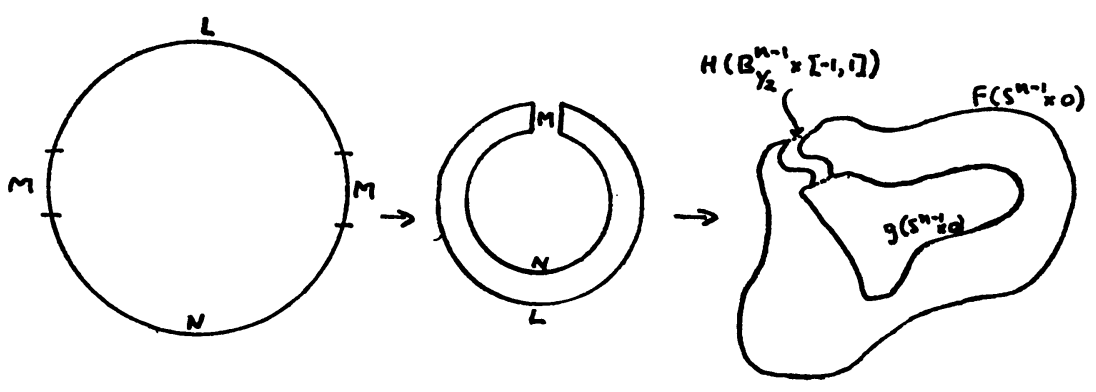

FIGURE 4

STEP 5. Bicollarings of $c(L), c(M)$, and $c(N)$ exist due to $a^{\prime}, H$ and $b^{\prime}$, because $k$ can be extended to a homeomorphism of $L \times[-1,1]$ $\rightarrow B_{i}^{n-1} \times[-1,1]$ in the natural way, as can $k^{\prime}$ and $k^{\prime \prime}$. Then, considering $\left(a^{\prime}\right)^{-1}$ of a neighborhood of $c(L \cap M)$ in $R^{n}$, Lemma 4 below gives a bicollaring of a neighborhood of $c(L \cap M)$, and similarly of $c(M \cap N)$. Thus every point of $c(L \cup M \cup N)=c\left(S^{n-1}\right)$ has a neighbor- 
hood with a bicollaring. Hence [1] $c\left(S^{n-1}\right)$ is bicollared. Then $c$ extends to $\hat{c}: B^{n} \rightarrow R^{n}$ (see [2]). We observe that $\hat{c}\left(B^{n}\right)=\bar{Z}$-int $B_{i}^{n-1}$ $\times[-1,1]$.

STEP 6. There exists a homeomorphism $d$ taking $S^{n-1} \times[-1,1]$ onto $B^{n} \bigcup_{k^{\prime}}\left(B_{3}^{n-1} \times[-1,1]\right)$ where $\partial B^{n}=L \cup M \cup N$ and $M$ is identified with $\partial B_{l}^{n-1} \times[-1,1]$ by $k^{\prime}$ (see Step 4$)$. Define a homeomorphism $A: S^{n-1} \times[-1,1] \rightarrow \bar{Z}$ by

$$
A(x, t)= \begin{cases}\hat{c} d(x, t) & \text { if } d(x, t) \in B^{n}, \\ h d(x, t) & \text { if } d(x, t) \in B_{1 / 2}^{n-1} \times[-1,1] .\end{cases}
$$

$A$ is well defined, for if $d(x, t) \in B^{n}$ and $d(x, t) \in B_{3}^{n-1} \times[-1,1]$, then $d(x, t) \in M \subset L \cup M \cup N=\partial B^{n}$, and for such a $d(x, t), \hat{c} d(x, t)$ $=H k^{\prime} d(x, t)=H d(x, t)$. This completes the proof of Theorem 2 .

Lemma 4. Let $C^{n-1} \subset R^{n}$ be the cylinder over (i.e. $x_{n} \geqq 0$ ) the unit $(n-2)$-sphere in $R^{n-1}$. Let $B^{n-1}$ be the unit $(n-1)$-ball in $R^{n-1}$. Then $C \cup\left(R^{n-1}-B^{n-1}\right)$ is bicollared.

Proof. Figure 5 indicates the proof for $n=2$, and the other dimensions are entirely analogous.

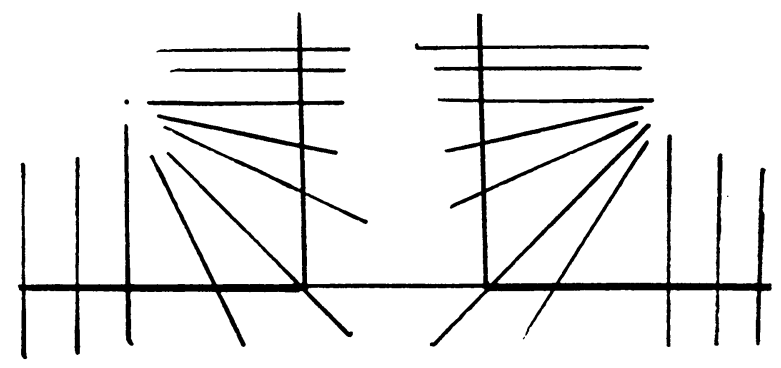

Figure 5

TheOREM 5. Let $f, g: S^{n-1} \times[-1,1] \rightarrow R^{n}$ be two imbeddings with disjoint images such that $f$ and $g$ are both somewhere planar. Then the imbedding $h$ of Theorem 2 exists, so the conclusion of Theorem 2 holds.

Proof. From the definition of somewhere planar, we have a neighborhood $N$ of $(p, q)$ in $S^{n-1} \times q$ and a homeomorphism $\pi: f(N) \rightarrow P$ where $\pi$ projects $f(N)$ in to $P$ along normals to $P$.

Instead of $P$ it is possible to choose any other $(n-1)$-plane parallel to $P$. Since we can also use a subneighborhood of $N$ (still containing $p$ ), there is no loss of generality in assuming that $\pi f(N)$ is a subset of 
$P$ lying in $\hat{Z}$ (the region between $f\left(S^{n-1} \times q\right)$ and $g\left(S^{n-1} \times q^{\prime}\right)$ ). See Figure 6.

Similarly (using primes) we have a neighborhood $N^{\prime}$ of $p^{\prime}$ in $S^{n-1} \times q^{\prime}$ such that $\pi^{\prime} g\left(N^{\prime}\right)$ is a subset of $P^{\prime}$ lying in $\hat{Z}$.

Let $C_{f}$ be the "cylinder" composed of line segments joining $x$ and $\pi(x)$ for all $x \in f(N)$. We may define $C_{o}$ in a similar way.

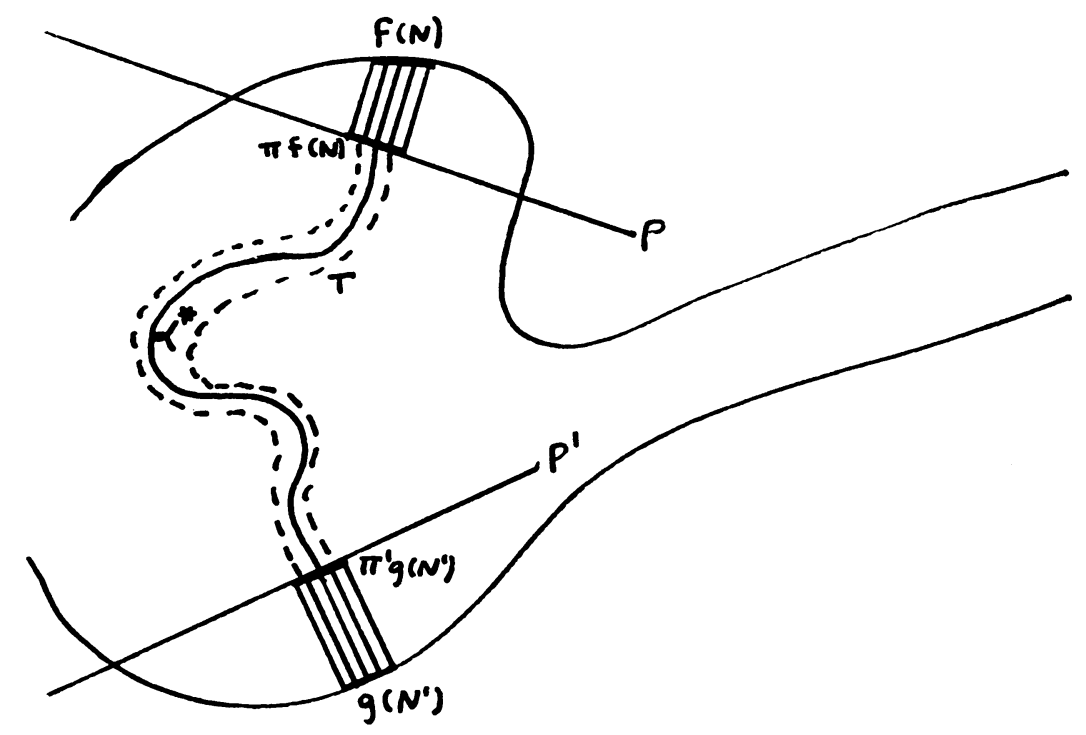

FiguRe 6

$\hat{Z}$ and hence $\hat{Z}-\left(C_{f} \cup C_{\theta}\right)$ are arcwise connected. Therefore it is possible to find an arc $\alpha$ in $\hat{Z}$, joining $\pi f(p)$ to $\pi^{\prime} g\left(p^{\prime}\right)$, perpendicular to $P$ near $\pi f(p)$, perpendicular to $P^{\prime}$ near $\pi^{\prime} g\left(p^{\prime}\right)$, and not intersecting $C_{f}-\pi f(p)$ and $C_{o}-\pi^{\prime} g\left(p^{\prime}\right)$. Let $\alpha^{*}$ be a differentiable approximation to $\alpha$ satisfying the same conditions as $\alpha$. $\alpha^{*}$ has a tubular neighborhood, i.e., there exists a differentiable imbedding $T: B^{n-1} \times\left[-\frac{1}{2}, \frac{1}{2}\right]$ $\rightarrow R^{n}$ so that $T\left(0 \times\left[-\frac{1}{2}, \frac{1}{2}\right]\right)=\alpha^{*}$. We may also require that $T\left(B^{n-1}\right.$ $\left.\times-\frac{1}{2}\right) \subset \pi f(N)$ and $T\left(B^{n-1} \times \frac{1}{2}\right) \subset \pi^{\prime} g\left(N^{\prime}\right)$, and that $T\left(B^{n-1}\right.$ $\left.\times\left(-\frac{1}{2}, \frac{1}{2}\right)\right) \subset \hat{Z}-\left(\bar{C}_{f} \cup \bar{C}_{g}\right)$.

We will obtain $h$ from $T, C_{f}$ and $C_{g}$. Define an imbedding $h$ : $B^{n-1} \times[-1,1] \rightarrow R^{n}$ by

$$
h(x, s)= \begin{cases}k_{f}(x, s) & \text { for }-1 \leqq s \leqq-\frac{1}{2}, \\ T(x, s) & \text { for }-\frac{1}{2}=s=\frac{1}{2} \\ k_{g}(x, s) & \text { for } \quad \frac{1}{2}=s=1\end{cases}
$$


where $k_{f}$ maps $\left(x \times\left[-1,-\frac{1}{2}\right]\right)$ linearly onto the line segment joining $f(y)$ to $\pi f(y)=T\left(x,-\frac{1}{2}\right)$ and $k_{0}$ maps $\left(x \times\left[\frac{1}{2}, 1\right]\right)$ linearly on to the line segment joining $\pi^{\prime} g(y)=T\left(x, \frac{1}{2}\right)$ to $g(y)$. See Figure 6 . This completes the proof.

The following theorem leads to a relatively weak condition under which an imbedding $f: S^{n-1} \times[-1,1] \rightarrow R^{n}$ is somewhere planar.

THEOREM 6. Let $h$ be a homeomorphism of $R^{n}$ into $R^{n}$. Let $h$ be differentiable with nonzero Jacobian at $p \in R^{n}$ and let $U$ be a neighborhood of $p$. Then there exists an isotopy $H_{t}: R^{n} \rightarrow R^{n}, t \in[0,1]$, satisfying

(1) $H_{0}=h$,

(2) $H_{t}=h$ on $R^{n}-U$ for all $t \in[0,1]$,

(3) there exists an open set $V, p \in V \subset U$, such that $H_{1}$ is differentiable on $V$.

Corollary 7. An imbedding $f: S^{n-1} \times[-1,1] \rightarrow R^{n}$ is somewhere planar if it is differentiable with nonzero Jacobian at $(p, q) \in S^{n-1}$ $\times(-1,1)$.

CoRollary 8. A homeomorphism $h: R^{n} \rightarrow R^{n}$ is stable if it is differentiable with nonzero Jacobian at $p \in R^{n}$.

Proof of Theorem 6. We can assume that $p=0$ and that $h(0)=0$. Denote by $D h$ the differential of $h$ at 0 , a nonsingular linear transformation. Let $k$ be a positive real number such that $B_{2 \mathbf{k}}^{n} \subset U$ and $D h\left(B_{2 \mathrm{k}}^{n}\right) \subset h(U)$.

We now define a radial homotopy $e_{t}: R^{n} \rightarrow R^{n}, t \in[0,1]$, as follows:

$$
e_{t}(x)= \begin{cases}x & \text { if } 2 k \leqq|x|, \\ \frac{2|x|-2 t k}{2|x|-|x| t} \cdot x & \text { if } t k \leqq|x| \leqq 2 k, \\ 0 & \text { if } 0 \leqq|x| \leqq t k .\end{cases}
$$

Let $H_{t}: R^{n} \rightarrow R^{n}$ be defined by

$$
H_{t}(x)= \begin{cases}D h e_{t}^{-1}(D h)^{-1} h e_{t}(x) & \text { if }|x|>t k \\ D h(x) & \text { if }|x| \leqq t k .\end{cases}
$$

Then conclusions (1), (2) and (3) of Theorem 6 are easily verified. The only difficulty is in proving that $H_{t}$ is continuous at $x$ when $|x|=t k$. We will indicate a proof (for details, see $[4, \S 3]$ ). Let $\lambda=\left\{x \in R^{n} \mid x_{1}>k, x_{2}=\cdots=x_{n}=0\right\}$. Then $e_{1}(\lambda)$ is the positive $x_{1}$ axis, $X_{1}$, and $h e_{1}(\lambda)$ has a tangent at 0 , namely $D h\left(X_{1}\right)$. Hence $(D h)^{-1} h e_{1}(\lambda)$ has a tangent at 0 , namely $X_{1}$, so $e_{1}^{-1}(D h)^{-1} h e_{1}(\lambda)$ con- 
verges to $(k, 0,0, \cdots, 0)$. This idea is used to show continuity of $H_{t}$ at $(k, 0,0, \cdots, 0)$, or in general, at any point of $S_{t k}^{n-1}$.

\section{REFERENCES}

1. M. Brown, Locally flat imbeddings of topological manifolds, Ann. of Math. 75 (1962), 331-341.

2. - A proof of the generalized Schoenflies theorem, Bull. Amer. Math. Soc. 66 (1960), 74-76.

3. M. Brown and H. Gluck, Stable structures on manifolds. I, II, III, Ann. of Math. 79 (1964), 1-58.

4. R. C. Kirby, Smoothing locally flat imbeddings, Doctoral Thesis, University of Chicago, Chicago, Ill., 1965.

5. R. Lashof, Problems in differential and algebraic topology, Ann. of Math. 81 (1965), 565-591.

6. B. Mazur, Relative neighborhoods and the theorems of Smale, Ann. of Math. 77 (1963), 232-249.

7. S. Smale, On the structure of manifolds, Amer. J. Math. 84 (1962), 387-399.

University of Chicago 